\title{
PRINCIPIO PRO PERSONA EN EL PROCESO FAMILIAR
}

\author{
PRO PERSONA PRINCIPLE IN THE FAMILY PROCESS
}

\author{
Fermín Contreras-Sánchez ${ }^{1} *$ \\ 1. Escuela Judicial del Estado de Tabasco, México. fermin_contreras_sanchez@hotmail.com \\ * Autor de correspondencia: Fermín Contreras-Sánchez, correo electrónico: fermin_contreras_sanchez@hotmail.com
}

\section{RESUMEN}

Este artículo se funda en el análisis de la omisión de aplicar el principio pro persona en los procesos familiares en Tabasco, en particular en los Juicios donde tienen intervención menores de edad, ya sea Guarda y Custodia, Controversia Familiar, Alimentos, Divorcios, Filiaciones, Paternidad en reconocimiento como en desconocimiento, entre otros, siendo éste un Derecho Humano (DH) consagrado en el artículo $1^{\circ}$ de la Constitución Política de los Estados Unidos Mexicanos (CPEUM) $\mathrm{y}$ en diversos Tratados Internacionales, Sentencias emitidas por los organismos internacionales, así como múltiples criterios jurisprudenciales pronunciados por nuestra Suprema Corte de Justicia de la Nación (SCJN). En el Estado de Tabasco, ante la problemática que se presenta en el núcleo familiar, por las constantes demandas que a diario se registran en los diversos Juzgados Familiares y Civiles, como es el caso de los Juicios de orden familiar antes citado, se hace necesario que los Jueces Familiares y Civiles, se obliguen a aplicar el principio pro persona y el principio pro niño o el interés superior de niñas, niños y adolescentes. Estos principios consisten en aplicar, en el sentido más amplio lo más benéfico a un menor de edad, en todo juicio en donde se encuentre involucrado, por lo que el juez en el caso concreto de la guarda y custodia al radicar o dar inicio a la demanda, debe decretar de oficio la convivencia provisional, escucha de menores de manera urgente, aunque las partes no lo soliciten ; escenario que actualmente los Jueces tanto Familiar y cómo Civil en nuestra entidad, no respetan ni aplican, siendo un derecho humano consagrado en los artículos $1^{\circ}$ párrafo segundo y $4^{\circ}$ párrafos noveno y décimo de nuestra Constitución.

Palabras claves: Derechos Humanos; Interés Superior del Menor; Juez; pro persona; Constitución Política de los Estados Unidos Mexicanos; Suprema Corte de Justicia de la Nación.

Cómo citar:

Contreras-Sánchez, Fermín. (2021). Principio pro persona en el proceso familiar. Revista de Investigaciones Universidad del Quindio, 33(S2), 71-82. https://doi.org/10.33975/riuq. vol33nS2.615 


\begin{abstract}
This article is based on the analysis of the failure to apply the pro persona principle in family proceedings in Tabasco, in particular in Trials where minors intervene, whether it is Guardianship and Custody, Family Controversy, Alimony, Divorces, Affiliations, Paternity in recognition as in ignorance, among others, this being a Human Right (DH) enshrined in Article 1 of the Political Constitution of the United Mexican States (CPEUM) and in various International Treaties, Sentences issued by international organizations, as well as multiple jurisprudential criteria pronounced by our Supreme Court of Justice of the Nation (SCJN). In the State of Tabasco, in view of the problems that arise in the family nucleus, due to the constant demands that are registered daily in the various Family and Civil Courts, as is the case of the aforementioned Family Trials, it is necessary that the Family and Civil Judges are bound to apply the pro person principle and the pro-child principle or the best interests of girls, boys and adolescents. These principles consist in applying, in the broadest sense, what is most beneficial to a minor, in any trial in which he is involved, so that the judge in the specific case of custody when filing or initiating the demand, must officially decree provisional coexistence, listen to minors urgently, even if the parties do not request it; scenario that currently the Judges, both Family and Civil in our entity, do not respect or apply, being a human right enshrined in articles 1 , second paragraph and 4 , ninth and tenth paragraphs of our Constitution.
\end{abstract}

Keywords: Human Rights; Superior Interest of the Minor; Judge; pro persona; Political Constitution of the United Mexican States; Supreme Court of Justice of the Nation.

\title{
INTRODUCCIÓN
}

La importancia del principio pro persona, está previsto a partir del año 2011 cuando se contempla en la Reforma Constitucional, el cual a la letra señala:

"Las normas relativas a los derechos humanos se interpretarán de conformidad con esta Constitución y con los tratados internacionales de la materia favoreciendo en todo tiempo a las personas la protección más amplia".

El principio pro persona, establece que todo juez o autoridad competente debe considerar y determinar aplicar la norma que más favorezca a la persona, así sea un ordenamiento normativo jurídico del Estado mexicano o instrumento internacional. Este principio sin duda conlleva un eficaz medio para que toda persona sin distinción haga valer y garantice sus derechos.

Otro de los factores fundamentales para poder implementar de manera efectiva el principio pro persona, es la difusión de los derechos humanos entre la población Tabasqueña y capacitar a jueces y magistrados.

Por lo que el caso concreto en estudio relativo a los Juicios ordinarios Civil de Guarda y Custodia de padres que se disputan la preferencia en la guarda y custodia de esos menores o en su caso la convivencia de un padre con los hijos, en nuestro Estado, la mayoría de los Jueces Familiares y 
Civiles, no cumplen con lo consagrado en los artículos $1^{\circ}$ párrafo segundo y $4^{\circ}$ párrafos noveno y décimo de nuestra Constitución, omitiendo la aplicación del principio pro persona y el principio del interés superior de la niñez, un derecho humano.

En ese sentido, el presente artículo con enfoque sobre el método exegético y hermenéutico jurídico, es abordado desde los criterios constitucionales, de tratados internacionales, Jurisprudencial, doctrinal y desde el análisis del principio pro persona y principio pro niños o principio del interés superior de niñas, niños y adolescentes, en particular de casos concretos en el proceso familiar en el Estado de Tabasco.

\section{1.- Reforma constitucional en materia de derechos humanos en México.}

En México, el año 2011 representó un cambio de paradigma, elevando a rango Constitucional los derechos humanos.

Los cambios en atención al principio pro persona, fueron:

\section{"En el artículo 1":}

Se transforma la denominación del Capítulo I, Título Primero para pasar de "De las garantías individuales" a "De los derechos humanos y sus garantías".

Todos derechos humanos contemplados en los tratados internacionales adquirieron mismo nivel que los dispuestos en la Carta Magna, "favoreciendo en todo tiempo a las personas la protección más amplia”.

En ese sentido, se señala que las autoridades con base a su competencia deben regirse bajo los principios (universalidad, interdependencia, progresividad e indivisibilidad) de derechos humanos, así como tener la obligación en derechos humanos de divulgar, difundir, proteger y garantizarlos y no hacer acción, omisión de violación de los derechos humanos.

En ese tenor, la aplicación e interpretación de la SCJN se ha visualizado a través de dos decisiones relevantes (2011 y 213).

2011. Expediente varios 912/2010, con resolución en 2011, se estableció el control de convencionalidad basado en un modelo difuso o hibrido.

Posteriormente, en 2013, la SCJN resuelve en contradicción de tesis 293/2011, la existencia del bloque de constitucionalidad.

Por ello, la aplicación de los derechos humanos se realiza mediante el principio pro persona:

"Derechos humanos contenidos en la Constitución y en los tratados internacionales. Constituyen el parámetro de control de regularidad constitucional, pero cuando en la Constitución haya una restricción expresa al ejercicio de aquéllos, se debe estar a lo que establece el texto constitucional. Décima Época, Pleno, Jurisprudencia, Gaceta del Semanario Judicial de la Federación, Libro 5, abril de 2014, Tomo I, Tesis: P./J. 20/2014 (10a.), Página: 202. La cual sostiene que "los derechos humanos, en su conjunto, constituyen el parámetro de control de regularidad constitucional, conforme al cual debe analizarse la validez de las normas y actos que forman parte del orden jurídico mexicano". 
Determinando que cuando se suscite o exista la contradicción prepondera la norma constitucional del Estado mexicano.

\section{2.- Principio pro niños o principio del interés superior de niñas, niños y adolescentes.}

El principio pro niños o principio del interés superior del menor, pretende o tiene como objetivo el acceso y garantía a todo menor de un estado de bienestar sin precariedad y necesidades; lo que invoca la interdependencia e indivisibilidad con la dignidad, respeto, integridad física, psicológica, moral y espiritual. Por lo que conlleva a considerarlo como un estado de bienestar para el niño.

Lo anterior, debido a que en su etapa de crecimiento y desarrollo debe tener la tutela de un adulto, por su estado de dependencia.

En ese sentido, toda autoridad, instancia jurisdiccional, competencia legislativa de todas las esferas tienen el deber y obligación de la protección más amplia del menor.

Los principios pro niños o principio del interés superior de la niñez, están consagrados en los siguientes marcos normativos en el orden internacional, regional, federal y local.

\section{I.- Marco normativo internacional:}

Los artículos 2.2. y 3.2 de la Convención sobre los Derechos del Niño (CDN) (1989), establecen:

\section{"Artículo 2}

2.- Los Estados Partes tomarán todas las medidas apropiadas para garantizar que el niño se vea protegido contra toda forma de discriminación o castigo por causa de la condición, las actividades, las opiniones expresadas o las creencias de sus padres, o sus tutores o de sus familiares.

\section{Artículo 3}

2. Los Estados Partes se comprometen a asegurar al niño la protección y el cuidado que sean necesarios para su bienestar, teniendo en cuenta los derechos y deberes de sus padres, tutores $u$ otras personas responsables de él ante la ley y, con ese fin, tomarán todas las medidas legislativas y administrativas adecuadas".

De igual manera el artículo 19 de la Convención Interamericana de Derechos Humanos (CIDH), instituye lo siguiente:

\section{"Derechos del Niño}

Todo niño tiene derecho a las medidas de protección que sus condiciones de menor requieren por parte de su familia, de la sociedad y del Estado”.

La Carta de las Naciones Unidas, en el artículo 1.1 señala:

“Los Propósitos de las Naciones Unidas son:

1.- Mantener la paz y la seguridad internacionales, y con tal fin: tomar medidas colectivas eficaces para prevenir y eliminar amenazas a la paz, y para suprimir actos de agresión u otros quebrantamientos de la paz; y lograr por medios pacificos, y de conformidad con los principios de la justicia y del derecho internacional, el ajuste o arreglo de controversias o situaciones 
internacionales susceptibles de conducir a quebrantamientos de la paz”.

De la misma forma el artículo 25.2 de la Declaración Universal de Derechos Humanos, establece:

"2. La maternidad y la infancia tienen derecho a cuidados y asistencia especiales. Todos los niños, nacidos de matrimonio o fuera de matrimonio, tienen derecho a igual protección social”.

E1 Pacto Internacional de Derechos Civiles y Políticos, en sus artículos 23.1. y 24.1 señalan:

\section{“Artículo 23}

1. La familia es el elemento natural y fundamental de la sociedad y tiene derecho a la protección de la sociedad y del Estado.

\section{Artículo 24}

1. Todo niño tiene derecho, sin discriminación alguna por motivos de raza, color, sexo, idioma, religión, origen nacional o social, posición económica o nacimiento, a las medidas de protección que su condición de menor requiere, tanto por parte de su familia como de la sociedady del Estado.

Este Artículo 10 del Pacto Internacional de Derechos Económicos, Sociales y Culturales, consagra el Derecho a la más amplia protección y asistencia a la familia (principio pro persona).

\section{II.- Marco normativo Regional:}

\section{Jurisprudencias de la CIDH, sobre casos concretos:}

Interés superior del niño/a:

Medidas y protección del Estado deben respetar el interés superior del niño/a

Opinión Consultiva OC-17/02.

53.- La protección de los niños en los instrumentos internacionales tiene como objetivo primordial el desarrollo armonioso de la personalidad de aquéllos y el disfrute de los derechos que les han sido reconocidos.

56. Esta constituido en la dignidad del menor resaltando velar acorde a las necesidades y requerimientos de los derechos del niño.

57. A este respecto, el principio 2 de la Declaración de los Derechos del Niño (1959) establece: "El desarrollo del niño debe de asegurarse desde lo físico, mental, moral, espiritual, social, saludable y normal con condiciones de dignidad y libertad.

58. El principio anterior se reitera y desarrolla en el artículo 3 de la Convención sobre los Derechos del Niño, que dispone: 1. El cual resalta la obligación de tribunales y autoridades competentes en preponderar el interés superior del menor a partir de un estado de bienestar.

60. Respecto a cuidados especiales hacia el menor debe de establecer medidas especiales de protección en cuanto a la incompetencia, inmadurez o debilidad del menor.

62. En correspondencia a lo anterior, atribuye responsabilidades y obligaciones compartidas del Estado, familia, comunidad y sociedad.

65. En cuanto a que haya limitación del derecho del menor, se debe prever y establecer mecanismos que aseguren los derechos del menor.

66. De lo anterior, el Estado debe establecer mecanismos preventivos y de atención. Prever mediante la familia total protección y atención del menor. 
En el mismo sentido general: Caso Bulacio Vs. Argentina. Fondo, Reparaciones y Costas. Sentencia de 18 de septiembre de 2003, párr. 134; Caso de los Hermanos Gómez Paquiyauri Vs. Perú. Fondo, Reparaciones y Costas. Sentencia de 8 de julio de 2004, párr. 163; Caso de la "Masacre de Mapiripán" Vs. Colombia. Sentencia de 2005.

Asunto L.M. respecto a Paraguay. Resolución de la Corte Interamericana de Derechos Humanos de 01 de julio de 2011

16. Precisamente por lo anterior, en vista de la importancia de los intereses en cuestión, como son en este asunto el derecho a la dignidad e integridad, el derecho a la identidad y el derecho a la protección de la familia, los procedimientos administrativos y judiciales que conciernen la protección de los derechos humanos de personas menores de edad, particularmente aquellos procesos judiciales relacionados con la adopción, la guarda y la custodia de niños y niñas que se encuentra en su primera infancia, debiendo ejercerse por parte de las autoridades de manera eficaz y eficiente. Lo anterior revela una necesidad de cautelar y de proteger el interés superior del niño, así como de garantizar los derechos en posible riesgo hasta tanto no se resuelva la controversia sobre el fondo y de asegurar el efecto útil de la eventual decisión que se adopte.

18. En atención a lo anterior, el mero transcurso del tiempo puede constituir un factor que favorece la creación de lazos con la familia tenedora o acogedora que, en una eventual decisión sobre los derechos del niño, podrían a su vez erigirse en el fundamento principal para no cambiar la situación actual del niño, principalmente debido a que se incrementa el riesgo de afectar seriamente el balance emocional y psicológico del mismo. En otros términos, el paso del tiempo se constituye inevitablemente en un elemento definitorio de vínculos afectivos que serían difíciles de revertir sin causar un daño al niño o niña. Esa situación comporta un riesgo que no sólo resulta inminente, sino que ya podría estar materializándose. Por ende, la mayor dilación en los procedimientos, independientemente de cualquier decisión sobre la determinación de sus derechos, puede determinar el carácter irreversible o irremediable de la situación de hecho actual y volver nugatoria y perjudicial para los intereses del niño L.M, cualquier decisión en contrario.

\section{III.- Marco normativo federal:}

El artículo $4^{\circ}$ párrafo noveno y décimo de CPEUM, nos señalan: “En todas las decisiones y actuaciones del Estado se velará y cumplirá con el principio del interés superior de la niñez, garantizando de manera plena sus derechos. Los niños y las niñas tienen derecho a la satisfacción de sus necesidades de alimentación, salud, educación y sano esparcimiento para su desarrollo integral. Este principio deberá guiar el diseño, ejecución, seguimiento y evaluación de las políticas públicas dirigidas a la niñez. (Párrafo adicionado DOF 18-03-1980 Reformado DOF 07-04-2000, 12-10-2011),

Los ascendientes, tutores y custodios tienen la obligación de preservar y exigir el cumplimiento de estos derechos y principios. Párrafo adicionado DOF 07-04-2000. Reformado DOF 12- 10-2011 El Estado otorgará facilidades a los particulares para que coadyuven al cumplimiento de los derechos de la niñez (Párrafo adicionado DOF 07-04-2000. Fe de erratas al párrafo DOF 12-04-2000)".

La Ley General de los Derechos de Niñas, Niños y Adolescentes, publicada en 2014 y Última reforma incorporada: 11 de enero de 2021, en su artículo 13 establece:

"Para efectos de la presente Ley son derechos de niñas, niños y adolescentes, de manera enunciativa más no limitativa, los siguientes: 
I.- Derecho a la vida, a la paz, a la supervivencia y al desarrollo;

IV. Derecho a vivir en familia;

VI. Derecho a no ser discriminado;

VII. Derecho a vivir en condiciones de bienestar y a un sano desarrollo integral;

VIII. Derecho a una vida libre de violencia y a la integridad personal”."

\section{IV.- Jurisprudencias de la Suprema Corte de Justicia de la Nación, sobre casos concretos:}

1).- "Registro digital: 2020401. Instancia: Segunda Sala. Décima Época. Materias(s): Constitucional. Tesis: 2a./J. 113/2019 (10a.). Fuente: Gaceta del Semanario Judicial de la Federación. Libro 69, agosto de 2019, Tomo III, página 2328. Tipo: Jurisprudencia

DERECHOS DE LAS NIÑAS, NIÑOS Y ADOLESCENTES. EL INTERÉS SUPERIOR DEL MENOR SE ERIGE COMO LA CONSIDERACIÓN PRIMORDIAL QUE DEBE DE ATENDERSE EN CUALQUIER DECISIÓN QUE LES AFECTE”.

2).- "Registro digital: 2021214. Instancia: Tribunales Colegiados de Circuito. Décima Época. Materias(s): Civil. Tesis: I.3o.C.398 C(10a.). Fuente: Gaceta del Semanario Judicial de la Federación. Libro 73, diciembre de 2019, Tomo II, página 1034. Tipo: Aislada CONTROVERSIAS DEL ORDEN FAMILIAR. CUANDO ALGUNA DE LAS PARTES INTERPONGA RECURSOS QUE LIMITEN O IMPIDAN EL EJERCICIO DE LOS DERECHOS DE NIÑOS, NIÑAS $Y$ ADOLESCENTES, LOS JUZGADORES DEBEN PRIVILEGIAR EL INTERÉS SUPERIOR DE ÉSTOS Y DICTAR LAS MEDIDAS CAUTELARES NECESARIAS PARA EVITAR QUE SE SIGAN AFECTANDO.".

3).- Registro digital: 2022082. Instancia: Tribunales Colegiados de Circuito. Décima Época. Materias(s): Civil. Tesis: XVII.1o.C.T.36 C (10a.). Fuente: Gaceta del Semanario Judicial de la Federación. Libro 78, septiembre de 2020, Tomo II, página 977. Tipo: Aislada

RÉGIMEN DE VISITAS Y CONVIVENCIAS DEL MENOR CON UNO DE SUS PROGENITORES, FRENTE A LA PANDEMIA GENERADA POR EL VIRUS SARS-COV 2 (COVID-19). ATENTO AL INTERÉS SUPERIOR DEL INFANTE, CORRESPONDE PRIVILEGIAR SU DERECHO A LA VIDA $Y$ A LA SALUD, SOBRE EL DERECHO A LA CONVIVENCIA CON AQUÉLLOS, POR ENDE, EL JUEZ DEBE PROVEER LAS MEDIDAS NECESARIAS PARA QUE ESTA ÚLTIMA SE EFECTÚE A DISTANCIA".

\section{V.- Marco normativo local:}

Además, en el ámbito local, la Ley de los Derechos de Niñas, Niños y Adolescentes del Estado de Tabasco, en los artículos $1^{\circ}$ y 2 que establecen:

"Artículo 1".- La presente Ley es de orden público, interés social y observancia general en el territorio del Estado de Tabasco y tiene por objeto:

"I.- Reconocer a niñas, niños y adolescentes como titulares de derechos humanos de conformidad con los principios establecidos en el artículo $1^{\circ}$ de la Constitución Política de los Estados Unidos Mexicanos y en el artículo 2 de la Constitución Política del Estado Libre y Soberano de Tabasco; II.- Garantizar en el Estado de Tabasco el pleno ejercicio, respeto, protección y promoción de los derechos humanos de niñas, niños y adolescentes conforme a lo establecido en la Constitución Política de los Estados Unidos Mexicanos, los tratados internacionales de la materia de los que el Estado Mexicano forma parte; en la Ley General de Derechos de Niñas, Niños y Adolescentes; la Constitución del Estado de Tabasco y en esta Ley.". 
"Artículo 2.- Para garantizar la protección de los derechos de niñas, niños y adolescentes, las autoridades estatales y municipales realizarán las acciones y tomarán las medidas necesarias, de conformidad con los principios establecidos en la Ley General y en la presente Ley.

Para tal efecto, deberán:

I.- Garantizar un enfoque integral, transversal y con perspectiva de derechos humanos en el diseño y la instrumentación de políticas públicas y programas estatales;

Todas las autoridades del Estado de Tabasco deberán considerar, de manera primordial, el interés superior de la niñez en la toma de decisiones, sobre una cuestión debatida que involucre derechos de niñas, niños y adolescentes.

Cuando se presenten diferentes interpretaciones, se elegirá la que satisfaga de manera más efectiva este principio rector. Cuando se tome una decisión que afecte a niñas, niños o adolescentes, en lo individual o en lo colectivo, se deberán evaluar y ponderar las posibles repercusiones que ello tenga, a fin de salvaguardar su interés superior y sus garantías procesales, en su caso."

1.3.- Vulneración del principio pro niño o principio del interés superior de niñas, niños y adolescentes, en el proceso de orden familiar en Tabasco, donde se encuentre involucrado un menor, ya sea Controversia Familiar, Alimentos, Divorcios, Filiaciones, Paternidad en reconocimiento como en desconocimiento y en el juicio ordinario civil de guarda y custodia de los padres con sus menores hijos.

El principio pro persona o pro homine y el principio pro niño o principio del interés superior de la niñez desde el orden normativo interno e internacional, es interpretativo y favorable a la persona. El caso concreto CINDY vs JORGE, se refiere a una controversia que se promueve en la VÍA INCIDENTAL DE MODIFICACIÓN DEL CONVENIO JUDICIAL DE FECHA 21 DE JUNIO DE 2018, derivado del expediente 376/2018, de divorcio incausado que se ventiló en el Juzgado Primero Familiar de Primera Instancia del Distrito Judicial de Centro, Tabasco, México, el cual fue aprobado y elevado a categoría de Cosa Juzgada, como si se tratase de Sentencia Ejecutoriada.

Dicho incidente obedece, en virtud, que el convenio judicial de referencia, se vulneraron los principios pro niño o principios del interés superior de niñas, niños y adolescentes, consagrados en las convenciones internacionales, jurisprudencias regionales, nuestra carta magna, jurisprudencia de la Corte Nacional, Ley Federal y Local, todas relativas a la protección de los derechos humanos y al interés superior de niñas, niño y adolescentes, que se mencionan en el apartado anterior, por los motivos siguientes:

En la Cláusula Segunda, del citado Convenio Judicial, se estableció lo siguiente: "CINDY y JORGE, convienen que después de divorciados, la guarda y custodia definitiva de la menor L.F.A.M., queda confiada en favor de su progenitor JORGE, y la guarda y custodia definitiva del menor J.A.A.M. queda a favor de su progenitora CINDY; ambos padres ejercerán la patria potestad de los referidos menores, en términos del numeral 4to. Constitucional, 2, 9, de la Convención de los Derechos del Niño, 23, 169, 405, 406, 407, 408, 417, 419, 422, 423,424, 429 y 453 del Código Civil y 492, 493 fracción II, 494 del Código de Procedimientos Civiles Vigentes”.

En la Cláusula Tercera, se fijó la forma en que se llevaría la convivencia con los menores de iniciales L.F.A.M. y J.A.A.M. entre otras cosas las siguientes:

"Acuerdan CINDY y JORGE, que después del divorcio, el régimen de convivencia al que se sujetarán los menores L.F. y J.A. de apellidos A.M., se llevará a efecto fuera del domicilio donde estos habitan con sus progenitores.

Al efecto, el menor J.A.A.M., convivirá con su progenitor de viernes a domingo de un fin de semana 
si y uno no; en un horario de seis de la tarde (06:00 p.m.), -viernes- hasta el-domingo- a las siete de la noche (07:00 p.m.).

De igual forma, la menor L.F.A.M., convivirá con su progenitora de viernes a domingo de un fin de semana si y uno no; en un horario de seis de la tarde (06:00 p.m.), -viernes- hasta el-domingo- a las siete de la noche (07:00 p.m.)"

Al entrar al análisis del caso concreto de estudio, consideró que los menores de iniciales L.F.A.M. y J.A.A.M., conviven muy poco tiempo juntos entre sí, así como con sus progenitores, ya que, sólo conviven los fines de semana, cuando la convivencia entre los menores de iniciales L.F.A.M. y J.A.A.M., debe ser constante y permanente, con la finalidad de crear lazos afectivos más fuertes entre los mismos, lo cual se lograría si los menores conviven a diario, es por ello que se solicitó ante Juez de la causa, la modificación de las cláusulas segunda y tercera del Convenio Judicial de fecha 21 de Junio de 2018, para efectos de que los menores de iniciales L.F.A.M. y J.A.A.M., puedan convivir permanentemente entre ellos, al solicitar la convivencia de quince (15) días naturales consecutivos con cada uno de sus progenitores, es decir, quince (15) días naturales consecutivos con la C. CINDY y quince (15) días naturales consecutivos con el C. JORGE.

Así también, al modificarse los párrafo segundo y tercero de la Cláusula Tercera del referido Convenio Judicial, en los términos antes citados, los progenitores tendrían una mayor convivencia con los menores de iniciales J.A.A.M y L.F.A.M., convivencia que no se limitaría a dos (2) fines de semana al mes, como se había convenido, sino que los menores de iniciales L.F.A.M. y J.A.A.M., convivirán de forma permanente entre sí, y convivirán quince (15) días naturales consecutivos con cada uno de sus progenitores. Dicha convivencia es con la finalidad de que los menores multicitados, crezcan tranquilos y sanos en todos los ámbitos personales, fortaleciéndose entre ellos los lazos de amor y respeto, ya que al estar en pleno ejercicio de la patria potestad, los progenitores conservan el derecho de convivir con sus hijos y la obligación de velar por su formación física, espiritual y moral, así también, es importante que se determine que cuando los menores estén conviviendo con uno de sus progenitores, el progenitor que no tenga en ese momento la guarda y custodia de los menores, podrá visitar o comunicarse por medios tecnológicos con los menores de iniciales L.F.A.M. y J.A.A.M., ya que el derecho de los menores de convivir continuamente con sus padres, no sólo permite que los padres en ejercicio de la patria potestad contribuyan a la protección, educación y formación integral de sus hijos, sino que además, permite que se formen relaciones estrechas entre padres e hijos, lo cual no sólo propicia relaciones paterno y materno filiales adecuadas, sino que además, esa relación necesariamente influye en la personalidad e identidad que en el futuro asumirán los menores, no obstante, en esta formación no sólo es importante la interacción que los menores tenga con sus padres, sino que además, resulta trascendente la interacción que éste tiene con el resto de los integrantes de su familia, incluida la ampliada en ambas líneas, es decir, la paterna y la materna, ya que ello no sólo contribuye a su formación, permitiendo identificarse como parte de un determinado grupo familiar. Por ello, en el desarrollo y formación de un menor, no sólo es importante que éste conviva estrechamente con sus padres, sino que, además, también son importantes las relaciones que mantenga con el resto de su grupo familiar, incluido el ampliado en ambas líneas, es decir, tanto el paterno como el materno, y por ello, al convivir los menores más días con sus progenitores, también, se propicia las relaciones personales con la familia ampliada, tanto paterna como materna.

Por todo lo anteriormente expuesto, el Juez Familiar que juzga, atendiendo el interés superior de los menores, debe sin justa causa realizar la modificación a las cláusulas del_Convenio Judicial de fecha 21 de junio de 2018, para permitir que la convivencia entre los menores de iniciales L.F.A.M. y 
J.A.A.M., sea constante y permanente en los términos y condiciones antes citados.

Por lo que el Juez de la Causa, al momento de resolver la controversia de guarda y custodia en el presente caso, debe aplicar los diversos criterios de la Suprema Corte de Justicia de la Nación, las jurisprudencias de la CIDH, lo consagrado en los artículos $1^{\circ}$ y $4^{\circ}$ de la CPEUM, las convenciones internacionales, leyes federales y estatales que se relaciona en este artículo para la protección más amplia de los derechos humanos de las niñas, niños y adolescentes y que aquí se reproducen para todos los efectos legales, atendiendo única y exclusivamente a la situación de guarda y custodia en la que la convivencia sea más benéfica para los menores, y se dejen de lado los intereses particulares de los propios progenitores, lo cual no ocurrió al aprobar y elevar a categoría de cosa juzgada como si se tratase de Sentencia Ejecutoriada el Convenio Judicial de fecha 21 de junio de 2018, que hoy se pretende modificar, toda vez que en su contenido no se veló por el derecho de los menores en cita, y que en el mismo se les negó la convivencia entre hermanos de forma continua y permanente, negándoles así el derecho crear lazos afectivos más fuertes entre los mismos, lo cual se lograría si los menores conviven a diario, vulnerando así los principios pro niño o principios del interés superior de niñas, niños y adolescentes, ya que por desconocimiento a los citados principios los impartidores de justicia no los aplican como es debido en los casos que resuelven en definitiva.

\section{CONCLUSIONES}

Después de haber realizado la investigación y desarrollo del presente artículo, utilizando el método interpretativo o hermenéutico jurídico, desde los criterios convencionales, constitucionales, Jurisprudencia regional y federal, doctrinal y de la aplicabilidad del principio pro persona y principio pro niños o principio del interés superior de niñas, niños y adolescentes, siendo éste un Derecho Humano (DH) consagrado en los artículo $1^{\mathrm{o}}$ y $4^{\mathrm{o}}$ de la Constitución Política de los Estados Unidos Mexicanos (CPEUM) y en diversos Tratados Internacionales, Sentencias emitidas por los organismos internacionales, así como múltiples criterios jurisprudenciales pronunciados por nuestra Suprema Corte de Justicia de la Nación (SCJN) misma que se transcriben en los apartados 1.1 y 1.2 de éste artículo, se concluye que las autoridades jurisdiccionales locales no aplican dichos principios a favor de las personas y en especial a favor del interés superior de niñas, niños y adolescentes, como en el caso concreto de estudio relativo a la guarda y Custodia de los padres con sus menores hijos antes citado, por lo que se hace inexcusable que los Jueces Familiares y Civiles, tengan como obligación ineludible aplicar el principio pro persona y apegarse al principio del interés superior de la niñez, por lo que al momento de resolver de forma provisional y posterior en forma definitiva deberán considerar lo más benéfico para los menores, ya que las niñas, niños y adolescentes se encuentran en proceso de formación y desarrollo, y por sus tipologías particulares dependen de las personas responsables de su cuidado para la realización de sus derechos; sin embargo, esta circunstancia puede llegar a limitar sus posibilidades de defender sus intereses.

Por ello, todos los órganos jurisdiccionales, autoridades administrativas y órganos legislativos (federal y locales) tienen la obligación de tomar en cuenta el interés superior como una consideración primordial y promover, respetar, proteger y garantizar los derechos de las personas menores de edad de conformidad con los principios de universalidad, interdependencia, indivisibili-dad y progresividad.

De esta manera se evitará en lo sucesivo, que se sigan causando daños emocionales, psicológicos y trastornos en el desarrollo humano de la niñez en Tabasco, para que se integren debidamente al núcleo familiar al que pertenecen. 


\section{REFERENCIAS}

1. Amaya Villarreal, Á. F. (2005). "El principio pro homine, interpretación extensiva vs consentimiento de los Estados . International Law. Revista Colombiana de Derecho Internacional, Bogotá, núm. 5 .

2. Astudillo, C. (2015). El bloque y el parámetro de constitucionalidad en la interpretación de la Suprema Cortede Justicia de la Nación. México : UNAM.

3. Caballero Ochoa, J. L. (2009). La incorporación de los tratados internacionales de los Derechos Humanos en España y México. México : Porrua, S.A.

4. Caballero Ochoa, J. L. (2014). , La interpretación conforme. El modelo constitucional ante los tratados internacionales sobre derechos humanos y el control de convencionalidad. México: Porrua, SA. .

5. Carbonell, M. (2008). El principio de proporcionalidad y la interpretación constitucional. México: Ministerios de Justicia y Derechos humanos, Quito.

6. Castañeda, M. (2015). El principio pro persona. Experiencias y expectativas. México : Comisión Nacional de los Derechos Humanos, UNAM.

7. Castilla, K. (2009). El principio pro persona en la administración de justicia. Revista Mexicana de Derecho Constitucional, Número 20.

8. Castilla, K. (2015). El principio pro persona a tres años de su inclusión en el texto constitucional federal. México : Tribunal Electoral del Poder Judicial de la Federación .

9. Ferrer Mac-Gregor, E. (2011). "Interpretación conforme y control difuso de convencionalidad, el nuevo paradigma para el juez mexicano. México: UNAM.

10. García Ramírez, S. (2014). Reparaciones por violación de derechos humanos, jurisprudencia interamericana. México: Porrua, S.A.

11. García Ramírez, S. y. (2013). La reforma constitucional sobre derechos humanos. México : Porrua, S.A., UNAM.

12. González, M. d. (2011). La evolución historica de Derechos Humanos en México. México: CNDH.

13. Hernández, M. C. (2018). El Principio Pro Persona ante la Ponderación de Derechos. México: Comisión Nacional de los Derechos Humanos CNDH.

14. Jorge, C. (1993). Derechos Humanos y Ombudsman. México : UNAM.

15. Medellín Urquiaga, X. (2013). Principio pro persona. México: CDHDF / SCJN / OACNDH.

16. Miguel, C. (2015). Los Derechos Humanos en México. México : Flores Editor.

17. Montermayor Romo de Vivar, C. (2002). La unifcación conceptual de los derechos humanos. México: Porrúa-Facultad de Derecho de la UNAM.

18. Ortega García, R. (2015). La jerarquía de los tratados internacionales sobre derechos humanos a la luz de la reforma constitucional del 10 de junio de 2011. México: Anuario Mexicano de Derecho Internacional.

19. Rosa, B. V. (s.f.). EL PRINCIPIO PRO PERSONA EN EL ESTADO CONSTITUCIONAL Y DEMOCRÁTICO DE DERECHO. Ciencia Jurídica Universidad de Guanajuato División de Derecho, Política y Gobierno Departamento de Derecho, 7, número 7.

20. Sergio, G. R. (2019). La Corte Interamericana de Derechos Humanos. México: Porrua, S.A.

21. Serrano, M. V. (2018). Derechos Humanos y la interpretación de la Corte en México, Ira Edic. México: Thomson Reuters.

22. Serrano, S. y. (2013). Los derechos en acción. Obligaciones y principios de derechos humanos. México: Flacso.

23. Vázquez, L. D. (2011). "Los principios de universalidad, interdependencia, indivisibilidad y progresividad. Apuntes para su aplicación práctica. México : Porrúa, S.A.

\section{Referencias complementarias}

24. Constitución Política de los Estados Unidos mexicanos.

25. Convención sobre los Derechos del Niño.

26. Convención Interamericana de Derechos Humanos (CIDH).

27. La Carta de las Naciones Unidas.

28. Declaración Universal de Derechos Humanos.

29. El Pacto Internacional de Derechos Civiles y Políticos. 
30. Pacto Internacional de Derechos Económicos, Sociales y Culturales.

31. Jurisprudencias de la CIDH, sobre casos concretos: Interés superior del niño/a.

32. Ley General de los Derechos de Niñas, Niños y Adolescentes.

33. Jurisprudencias de la Suprema Corte de Justicia de la Nación, sobre casos concretos.

34. Ley de los Derechos de Niñas, Niños y Adolescentes del Estado de Tabasco. 